Rapid Reviews COVID-19

\title{
Review 1: "Wastewater Surveillance of SARS-CoV- 2 across 40 U.S. states"
}

\author{
Rosa Pintó ${ }^{1}$ \\ ${ }^{\mathbf{1}}$ Full Professor, University of Barcelona: Universitat de Barcelona, Genetics, Microbiology and \\ Statistics, Spain
}

Published on: Jun 16, 2021

License: Creative Commons Attribution 4.0 International License (CC-BY 4.0). 


\section{$\underline{\text { RR:C19 Evidence Scale rating by reviewer: }}$}

- Potentially informative. The main claims made are not strongly justified by the methods and data, but may yield some insight. The results and conclusions of the study may resemble those from the hypothetical ideal study, but there is substantial room for doubt. Decision-makers should consider this evidence only with a thorough understanding of its weaknesses, alongside other evidence and theory. Decisionmakers should not consider this actionable, unless the weaknesses are clearly understood and there is other theory and evidence to further support it.

*****************************************

\section{Review:}

This manuscript, although containing a big amount of data, fails to clearly prove the claims included, mainly due to flaws in the methodological process.

The volume of wastewater sampled is too small to ensure the detection of genome copies of SARS-CoV-2 in samples with low levels of viruses. Two different water concentration methods are applied, which use $40 \mathrm{ml}$ and $15 \mathrm{ml}$ of wastewater, respectively. These volumes will permit genome detection only in highly contaminated samples, which may explain why only $49 \%$ of the samples were detected to be positive for SARS-CoV-2 during the first wave of the pandemic. Additionally, the description provided does not precisely reflect the volume of the water concentrate used for RNA extraction or the volume of RNA used for the RT-qPCR. The limit of quantification is also missing.

Another critical issue is the lack of a process control coronavirus for the estimation of the efficiency of the concentration procedure, which is required for the detection of SARS-CoV-2 concentration failures.

Additionally, there is no information on the confirmation method used. It would be advisable to use at least two RTqPCR targets, preferably from different genomic regions. In the methods sections, it is described that samples were analyzed for the CDC N1 or N2 targets. Fig 1, Fig S1 and Fig S2 legends do not contain information on the target used for the SARS-CoV-2 quantification. For instance, it is not clear if all data points included in the figures were quantified using the same target. Also, it is not described if all points were confirmed with a second target. 
Several claims on correlations between viral loads in wastewater samples and cases in the population are given, but no methods on the statistical analysis employed are provided. Additionally, assessments looking for the relationship of SARS-CoV-2 genome copy numbers in wastewater samples and cases in the population were performed using clinical data reported for the day on which the samples were obtained. However, SARS-CoV-2 infected patients shed viruses in feces for a period extending up to 7 to 21 days post-infection. Accordingly, the conclusions presented may not be accurate.

While figures are generally well presented, results in the text are sometimes confusing.

Literature is not extensive and, while many references are missing, some references that have been cited as preprints have already been published.

The flaws in the methods are significant, bringing doubts on the accuracy of the claims and conclusions. 